\title{
CIRED 2021: Bericht über die Session 3 - Operation, Control, and Protection
}

\author{
A. Abart OVE, I. Hübl OVE \\ angenommen am 19. Oktober 2021, online publiziert am 8. November 2021 \\ (c) Springer-Verlag GmbH Austria, ein Teil von Springer Nature 2021
}

Für die Session 3 der CIRED 2021 wurden zu den Themen Betrieb, Steuerung und Schutz rund 280 Abstracts eingereicht und davon 185 zur Einreichung eines „Full Paper" eingeladen. Insgesamt erreichten schließlich 147 Beiträge (zehn aus Österreich) das Team der Session 3 unter Leitung von Chairman Prof. Markus Zdrallek (Bergische Universität Wuppertal) sowie Carsten Boese (Erlanger Stadtwerke AG), Ignaz Hübel (ehem. KNG-Kärnten Netz GmbH) und Andreas Abart (Netz OÖ) als Rapporteure. Für die vier Blöcke der MainSession wurden 24 Beiträge zum Vortrag ausgewählt, neun im „Research and Innovation Forum" vorgestellt und die Mehrheit der verbleibenden Beiträge in neun Poster-Sessions präsentiert. Einen Überblick über alle eingereichten Full Paper bietet der Special Report der Session 3, welcher unter CIRED 2021 (https://www.cired2021.org/) zum Download bereitgestellt ist.

Im Themenfeld Betrieb waren unter 46 Beiträgen, wie auch in den vergangenen Jahren, interessante Beiträge im Bereich Inspektion, Instandhaltung und Ausbildung mit zunehmend praxisnahen Lösungen mit "Augmented Reality". Anwendungen der Künstlichen Intelligenz wurden im Bereich der Prognosen, der Fehleranalyse und der Zustandsbewertung aus Bildern präsentiert. Die DSO/TSOThemen betreffend war wieder das Thema der wachsenden kapazitiven Blindleistung während Schwachlast dominierend. Weiters wurde über die künftige Koordination für die Nutzung der Flexibilität aber auch über die Netzstabilität und Regelung bei sinkendem Anteil rotierender Massen berichtet. Dass im realen Betrieb durch das Zusammenwirken verschiedener Komponenten unerwartet Probleme auftreten, zeigte auf eindrucksvolle Weise ein Bericht über Tests an der Notstromversorgung eines Eisenbahntunnels.

Im Themenbereich Netzsteuerung mit insgesamt 69 Beiträgen war die Automatisierung der Mittelspannungsnetze vorherrschend. Zur Automatisierung der Niederspannungsnetze zeigt ein Blick auf vergangene Konferenzen einen stetigen Zuwachs. Dabei wächst vor allem auch der Anteil an Berichten über die Implementierung, während früher vor allem Pilotanwendungen dargestellt wurden. Die zunehmende Verfügbarkeit von Echtzeitdaten und Steuerungsmöglichkeiten, zum Teil auch über Smart Meter-Systeme, ergeben ein wachsendes Interesse an Flexibilität im Verteilnetz.

Unter den 35 Einreichungen zum Thema Schutz wurde über aktuelle Erkenntnisse zur Fehlerortung, dem Erdschlussverhalten, insbesondere in gelöschten Netzen, sowie über Schutzanwendungen in der Praxis und Algorithmen berichtet. Die immer besser werdenden Verfahren zur raschen und zuverlässigen Erkennung und Lokalisierung unter Verwendung moderner Kommunikationstechnologien, wie z. B. 5G, standen im Mittelpunkt der Diskussionen. Dabei zeigt sich auch wachsendes Interesse bezüglich der Anforderungen an die IT Security. Besonders erwähnenswert sind auch Beiträge über Feldtests und Untersuchungen in Mittel- und Niederspannungsnetzen.

In der Session 3 gab es drei Round Tables (RT7, RT8 und RT10) zu den Themen "Communication in Substations", "Monitoring and Control of LV Networks" und "Microgrids: Towards a Symbiosis between DSO and Microgrids?". Der RT7 befasste sich mit der Redun- danz von Kommunikationseinrichtungen in Umspannwerken. Anlagenkonfigurationen mit IEC-61850-Prozessbus wurden ausführlich diskutiert. Fred Steinhauser und drei Panelists zeigten, dass Netzwerkredundanz ein "Muss" in Anlagen mit redundantem Schutz ist. Beim RT8 wurden die Ergebnisse der CIRED-Arbeitsgruppe WG 2019-5 präsentiert und diskutiert. Dabei wurden der aktuelle Stand ebenso wie Zukunftsperspektiven der Überwachung und Steuerung von Niederspannungsnetzen beleuchtet. Der Fokus beim Monitoring von Niederspannungsnetzen liegt in der Verwendung von Smart Meter-Daten und Funktionen. Dazu gab es in der Session 4 einen Round Table RT11 „New Role of Smart Metering in Grid Planning, Control, and Operation" über die Ergebnisse einer weiteren CIREDArbeitsgruppe WG 2018-5. Die Berichte beider Arbeitsgruppen befanden sich zum Zeitpunkt der Konferenz in Fertigstellung. Der Round Table RT10 betreffend Microgrids befasste sich mit den Ergebnissen der CIRED-Arbeitsgruppen WG 2019-2 und 2018-3 über Geschäftsmodelle beziehungsweise über Betrieb mit Verteilnetzanbindung und als Insel (die Berichte sind online verfügbar).

Im Research and Innovation Forum (RIF) wurden neun Beiträge von Universitäten und Forschungseinrichtungen zu breit gestreuten Themen über alle Spannungsebenen präsentiert.

In allen Bereichen der Session 3 war die Qualität und das Engagement der Autor/innen, aber auch der Konferenzorganisation sehr hoch. Trotz des Online-Formats konnte die Möglichkeit zur Diskussion in den Live-Sessions genutzt werden. Viele interessante Inhalte der CIRED-2021-Session 3 werden wohl noch beim Workshop 2022 und der CIRED 2023 im persönlichen Austausch unter den Teilnehmer/innen diskutiert werden.

Hinweis des Verlags Der Verlag bleibt in Hinblick auf geografische Zuordnungen und Gebietsbezeichnungen in veröffentlichten Karten und Institutsadressen neutral.

26. Internationale Konferenz über elektrische Verteilnetze, virtuell, 20. bis 23. Septembe 2021.

Abart, Andreas, Netz Oberösterreich GmbH, Neubauzeile 99, 4030 Linz, Österreich (E-Mail: andreas.abart@netzooe.at); Hübl, Ignaz, Glödnitz, Österreich 